\title{
Tocilizumab treatment of therapy refractory thoracic aortitis in a patient with retroperitoneal fibrosis
}

\author{
Josephine BM Christ ${ }^{1 *}$, Detlef Moka ${ }^{2}$ and Johannes Strunk ${ }^{1}$ \\ ${ }^{1}$ Department of Rheumatology, Hospital Porz am Rhein, Urbacher Weg 19, 51149 Cologne, Germany \\ ${ }^{2}$ Center for Nuclear Medicine, Henricistr. 40, 45136 Essen, Germany
}

\begin{abstract}
In this report, we present the case of a patient with retroperitoneal fibrosis and aortitis.

Most authors suggest glucocorticoid treatment as first-line therapy. Further optimal steroid-sparing medication has not been established. Over the years, our patient was treated with mycophenolate mofetil, methotrexate, infliximab, etanercept, azathioprine and several steroid-pulse therapies.

Despite of this wide immunosuppressive treatment regular fluor-18-fluorodeoxyglucose positron emission computed tomography scans revealed refractory aortitis of the dilated ascending aorta.

Since a growing number of reports support the efficacy of B cell depletion with rituximab in patients with retroperitoneal fibrosis and aortitis, we started medication with rituximab. After nine month of treatment a follow-up fluor-18-fluorodeoxyglucose positron emission computed tomography scan still showed unaffected aortitis.

Since the aortitis was refractory to previous immunosuppressive drugs we decided to start medication with tocilizumab. This seemed feasible, since several studies indicated that tocilizumab may be a useful therapy option in patients with inflammatory aortitis and in patients with large vessel vasculitis featuring a positive response upon treatment with tocilizumab.

Six months after the last cycle of rituximab, we initiated weekly subcutaneous treatment with 162 mg tocilizumab. The most recent fluor-18-fluorodeoxyglucose positron emission computed tomography scan after six months of treatment revealed a significant decrease in fluorodeoxyglucose uptake at both the thoracic and abdominal aorta following tocilizumab therapy.

To avoid complications like aneurysm or dissection, effective treatment of aortitis related to retroperitoneal fibrosis is necessary. Optimal treatment has not been established, yet. Here, tocilizumab was effective to treat therapy refractory retroperitoneal fibrosis - related aortitis.
\end{abstract}

\section{Case report}

In 2005, a 53-year-old woman developed abdominal pains and a subileus. A computed tomography (CT) revealed retroperitoneal fibrosis (RF) encasing the distal abdominal aorta and iliac arteries. Both ureters were embedded in the retroperitoneal tissue, leading to a ureteral obstruction and hydronephrosis of the right kidney. Blood levels of C-reactive protein (CRP) were $2.3 \mathrm{mg} / \mathrm{dl}$ (reference $0.5 \mathrm{mg} / \mathrm{dl}$ ), erythrocyte sedimentation rate was $58 / 95 \mathrm{~mm} / \mathrm{h}$. Double-J stents were inserted in both ureters and surgery was performed for retroperitoneal resection. Histologically, the resected tissue featured inflammatory fibrosis associated with lymphoplasmacytic infiltration and neutrophil and eosinophil leucocytes, thus confirming the diagnosis of RF.

$\mathrm{RF}$ is a rare condition characterized by the replacement of normal tissue by an inflammatory and fibrous retroperitoneal tissue mass often entrapping the abdominal aorta and ureters $[1,2]$. RF may be idiopathic or secondary to other causes [2].

First-line therapy of RF is glucocorticoid treatment [2]. Accordingly, the patient received intravenous steroid pulse therapy, followed by oral steroid medication. Thereafter, steroidsparing medication with mycophenolate mofetil (MMF) was started.

In 2007, the patient received a fluor-18-fluorodeoxyglucose positron emission CT (F-18-FDG-PET/CT) scan. Beside the known RF with fluorodeoxyglucose (FDG) uptake at a standardized uptake value (SUV) of 4.5, the F-18-FDG-PET/CT scan revealed FDG uptake by the thoracic ascending aorta at a SUV of 3.9. Arteriosclerotic plaques were not obvious by the F-18-FDG-PET/CT scan and further exclusion of arteriosclerosis revealed clinical inconspicuous findings.

Recently, RF was reported to be associated occasionally with inflammatory aortitis $[3,4]$. Hence, without indications for a manifest arteriosclerosis the findings by the F-18-FDG-PET/CT scan were classified an aortic vasculitis in the context of RF.

The dosage of MMF was increased and the patient again received steroid pulse therapy.

Due to an allergic reaction, treatment was switched to azathioprine in 2008. Since a F-18-FDG-PET/CT scan in 2009 showed improvement

${ }^{\star}$ Correspondence to: Josephine BM Christ, Department of Rheumatology, Hospital Porz am Rhein, Urbacher Weg 19, 51149 Cologne, Germany, E-mail: josephine.b.m.christ@gmail.com

Key words: aortitis, autoimmune diseases, retroperitoneal fibrosis, radionuclide imaging, vasculitis

Received: March 17, 2020; Accepted: April 03, 2020; Published: April 13, 2020 
of the FDG uptake by the thoracic aorta, azathioprine was stopped, and the patient received no further immunosuppressant.

In the clinical course, the patient developed back pain between the shoulder blades in 2012.

A follow-up F-18-FDG-PET/CT scan showed disease progression displaying an increased FDG uptake by the ascending aorta and the aortic root with increasing dilatation (SUV 8.46). The abdominal manifestation showed only low inflammatory activity (SUV 2.8).

The four years following, the patient was treated with methotrexate, infliximab, etanercept and again azathioprine, and received several steroid pulse therapies. Within this time period, regular F-18-FDG$\mathrm{PET} / \mathrm{CT}$ scans showed no improvement with refractory aortitis. Upon persistent inflammatory activity of the dilated ascending aorta at a SUV of 7.65 as shown in a F-18-FDG-PET/CT scan in 2016, attending physicians presumably facing no therapeutic alternatives, started MMF again at $720 \mathrm{mg}$ per day.

To evaluate further therapeutic options, the now 65-year-old patient visited our department of Rheumatology in March 2017. Laboratory tests showed negative CRP $(<0.4 \mathrm{mg} / \mathrm{dl}$, reference $0.5 \mathrm{mg} /$ dl) and repeatedly normal IgG4 levels of $32.9 \mathrm{mg} / \mathrm{dl}$ (reference range $5.2-125 \mathrm{mg} / \mathrm{dl})$.

First, under good drug compatibility, we increased MMF to 1440 mg per day. However, the next F-18-FDG-PET/CT scan revealed ineffectiveness with constant FDG uptake (SUV 7.26) by the ascending aorta.

Emerging evidence supported the efficacy of B cell depletion with rituximab (RTX) in patients suffering from RF and aortitis [1,5]. Therefore, we started RTX medication with two intravenous doses of $1000 \mathrm{mg}$ at intervals of two weeks. The patient received the first cycle of RTX in June and July 2017, the second in December 2017. Despite of the treatment with RTX, the follow-up F-18-FDG-PET/CT scan in February 2018 revealed refractory aortitis (Figure 1A) with a persistent high FDG uptake by the ascending aorta (SUV 6.0).

Several studies indicate that tocilizumab (TCZ) might be useful for therapy of inflammatory aortitis $[4,6]$. Patients with large vessel vasculitis profited from treatment with TCZ [7]. Loricera et al. reported of a patient with aortitis and RF, who was successfully treated with TCZ [4]. However, this patient did not receive any other previous conventional immunosuppressive drugs. Vaglio et al. also described the successful use of TCZ in patients with RF [6].

Therefore, since the aortitis of our patient was refractory to previous immunosuppressive drugs including TNF-alpha inhibitors, corticosteroids and RTX, we decided to start medication with TCZ. Six months after the last cycle of RTX, we weekly administered 162 mg TCZ subcutaneously. After two months of treatment, the patient experienced improvement of her back pain.

The latest F-18-FDG-PET/CT scan in November 2018 revealed a significant decrease in FDG uptake at both the thoracic aorta (SUV 2.0) and the abdominal manifestation (SUV 1.9) following TCZ therapy (Figure 1B).

To avoid complications like aneurysm or dissection, effective treatment of aortitis related to RF is necessary. Optimal treatment has not been established, yet. Here, TCZ was effective to treat therapy refractory RF-related aortitis. Future studies must reveal whether treatment with TCZ may generally be effective in patients with RF and aortitis.
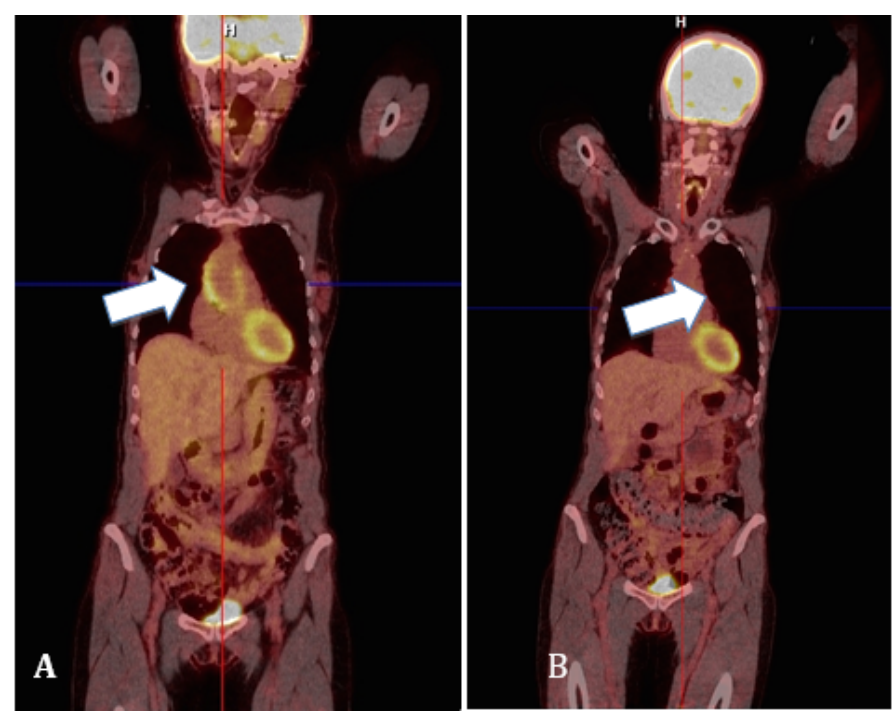

Figure 1. Fluor-18-fluorodeoxyglucose positron emission computed tomography images of the patient after rituximab and tocilizumab treatment. A: Aortitis with fluorodeoxyglucose (FDG) uptake by the thoracic ascending aorta after nine month of rituximab treatment. B: Fade of FDG uptake after six months of tocilizumab therapy

\section{Funding information}

Josephine Christ, Detlef Moka and Johannes Strunk declare that they have no conflict of interest and that they received no specific funding for this work.

\section{References}

1. Vaglio A, Maritati F (2016) Idiopathic Retroperitoneal Fibrosis. J Am Soc Nephrol 27 1880-1889 [Crossref]

2. Khosroshahi A, Carruthers MN, Stone JH, Shinagare S, Sainani N, et al. (2013) Rethinking Ormond's disease: "idiopathic" retroperitoneal fibrosis in the era of IgG4related disease. Medicine (Baltimore) 92: 82-91. [Crossref]

3. Stone JR (2011) Aortitis, periaortitis, and retroperitoneal fibrosis, as manifestations of IgG4-related systemic disease. Curr Opin Rheumatol 23: 88-94. [Crossref]

4. Loricera J, Blanco R, Castañeda S, Humbría A, Ortego-Centeno N, et al. (2014) Tocilizumab in refractory aortitis: study on 16 patients and literature review. Clin Exp Rheumatol 32: 79-89. [Crossref]

5. Wallwork R, Wallace Z, Perugino C, Sharma A, Stone JH (2018) Rituximab for idiopathic and IgG4-related retroperitoneal fibrosis. Medicine (Baltimore) 97: e12631. [Crossref]

6. Vaglio A, Catanoso MG, Spaggiari L, Magnani L, Pipitone N, et al. (2013) Interleukin-6 as an inflammatory mediator and target of therapy in chronic periaortitis. Arthritis Rheum 65: 2469-2475. [Crossref]

7. Unizony S, Stone JH, Stone JR (2013) New treatment strategies in large-vessel vasculitis. Curr Opin Rheumatol 25: 3-9. [Crossref]

Copyright: (C2020 Christ JBM. This is an open-access article distributed under the terms of the Creative Commons Attribution License, which permits unrestricted use, distribution, and reproduction in any medium, provided the original author and source are credited. 